\title{
New additional scoring formula on the Pathological Features in Stage I Lung Adenocarcinoma Patients: Impact on Survival
}

\author{
Shilei Zhao ${ }^{1}$, Fengzhou $\mathrm{Li}^{1}$, Xin Guo ${ }^{2}$, Tao Guo ${ }^{1}$, Ken-ichi Mizutani², Sohsuke Yamada ${ }^{2}$, Chundong Gu ${ }^{\circledR}$ \\ and Hidetaka Uramoto ${ }^{3 凶}$ \\ 1. Department of Thoracic Surgery, the First Affiliated Hospital of Dalian Medical University, Dalian, Liaoning, China. \\ 2. Department of Pathology and Laboratory Medicine, Kanazawa Medical University, Kanazawa, Ishikawa, Japan. \\ 3. Department of Thoracic Surgery, Kanazawa Medical University, Kanazawa, Ishikawa, Japan. \\ $\square$ Corresponding authors: Chundong Gu. Department of Thoracic Surgery, the First Affiliated Hospital of Dalian Medical University, Zhongshan road 222\#, \\ Dalian 116011, China; Hidetaka Uramoto. Department of Thoracic Surgery, Kanazawa Medical University, 1-1 Uchinada, Ishikawa 920-0265, Japan. Tel.: +86 411 \\ 83635963 ext 2061/+81-76-286-2211 ext 5721; Fax: +86-411-83622844/+81-76-286-1207; E-mails: guchundong@dmu.edu.cn. or hidetaka@kanazawa-med.ac.jp. \\ () The author(s). This is an open access article distributed under the terms of the Creative Commons Attribution License (https://creativecommons.org/licenses/by/4.0/). \\ See http://ivyspring.com/terms for full terms and conditions.
}

Received: 2020.02.17; Accepted: 2020.05.26; Published: 2020.07.19

\begin{abstract}
Background: Histological heterogeneity of lung adenocarcinoma may result in different prognosis among patients with the same TNM pathological stage. However, no objective evaluation system of lung adenocarcinoma based on pathological features has been widely accepted for assessing the prognosis.

Methods: We retrospectively analyzed 179 patients with stage I lung adenocarcinoma after complete surgical resection. The pathological classification was according to the IASLC/ATS/ERS adenocarcinoma classifications, and the detailed abundance ratio using HE staining of primary tumor specimens was recorded. A new additional scoring formula on the pathological features (ASP) was established. The association of the ASP score with the patients' survival was examined.

Results: The ASP scoring was significantly associated with smoking history $(p=0.004)$, lymphatic vessel invasion $(p<0.001)$, vascular invasion, differentiation $(p<0.001)$ and $\mathrm{Ki67}(p<0.001)$. The patients in the high-ASP-score group tended to have vascular invasion (odds ratio [OR]: 1.637, 95\% confidence interval [Cl]: 1.923-13.745, $\mathrm{p}=0.001$ ) and high Ki67 expression (OR: $2.625,95 \% \mathrm{Cl}: 1.328-5.190, \mathrm{p}=0.006$ ) by logistic regression analyses. The prognosis differed significantly in the Kaplan-Meier survival curves, and the 5-year survival rates in the low and high ASP score groups were $97.8 \%$ and $89.6 \%$, respectively $(p=0.018)$. Based on the univariate analysis, female (OR: $0.111,95 \% \mathrm{Cl}: 0.014-0.906, p=0.040)$, long smoking history (OR: 7.250, 95\%Cl: 1.452-36.195, $\mathrm{p}=0.016$ ), poor differentiation characteristics correlation (OR: 12.691, 95\% Cl: 1.557-103.453, $\mathrm{p}=0.018$ ), and high ASP score (OR: $5.788,95 \% \mathrm{Cl}$ : 1.138-29.423, $\mathrm{p}=0.034$ ) were shown to be independently associated with an unfavorable prognosis.

Conclusion: The ASP score can effectively screen high-risk patients for complete surgical resection of stage I lung adenocarcinoma.
\end{abstract}

Key words: Prognosis; Pathological classification; Lung adenocarcinoma, Heterogeneity

\section{Introduction}

In recent years, adenocarcinoma has ranked as the most important pathological type of lung cancer. As such, early screening programs for high-risk populations have been developed, and its incidence is increasing gradually year by year around the world $[1,2,3]$. Although the diagnosis and treatment of lung cancer continues to progress, resulting in an improved prognosis [4,5] lung adenocarcinoma, as a representative of tumor heterogeneity, has a poor prognosis due to its characteristics of early metastasis and recurrence [6,7]. Even in stage I lung adenocarcinoma, the five-year survival rate is still 
$70 \%$, and $30 \%$ high-risk patients tend to experience tumor progression $[8,9,10]$. Therefore, how to differentiate the high-risk group from stage I lung adenocarcinoma patients after surgery has become an urgent clinical problem to be solved.

In clinical practice, evidence supporting the further treatment of patients after complete surgical resection of stage I lung adenocarcinoma is lacking [11]. However, we frequently encounter various pathological subtypes from the same lung adenocarcinoma lesion, and its proportion in tumor tissue is also discrepant, due to the tumor's inherent histological heterogeneity. With the introduction and subsequent popularization of the International Association for the Study of Lung Cancer (IASLC), the American Thoracic Society (ATS), and the European Respiratory Society (ERS) lung adenocarcinoma classification systems since 2011 [12], most literature has shown that the prognosis of stage I lung adenocarcinoma patients differs based on the predominant pathological subtype $[13,14,15]$. However, despite these findings, detailed information on pathological mixed subtypes and the proportion of different morphological structures in the focus of adenocarcinoma has not been attracted sufficient attention. This may be the breakthrough point for identifying high-risk patients from these who the same pathological TNM staging, especially in early lung cancer.

At present, no objective system for evaluating lung adenocarcinoma has been widely accepted, in stark contrast to the situation with other tumors, such as breast, prostate, and kidney cancers $[16,17,18]$. Therefore, the present study explored a novel pathological risk scoring system of stage I lung adenocarcinoma based on pathological subtypes and the abundance ratio using hematoxylin-eosin staining. We also examined the frequency and clinical significance of additional scoring formula on the pathological features (ASP) in a retrospective series of 179 patients resected for adenocarcinoma of the lung and analyzed its impact on patients' prognosis. Our results indicate that ASP could facilitate the differentiation of high-risk patients from lower-risk ones and leading to appropriate early postoperative intervention.

\section{Methods}

\section{Patients and follow-up}

A total of 244 consecutive patients who underwent radical surgery of the primary tumor and systematic lymph node dissection or sampling at the Department of Thoracic Surgery of Kanazawa Medical University from January 2005 to December
2015 were included in this study. Of these, 179 patients (median age: 68.7 years old; range: 37-83 years old) were confirmed to have stage I lung adenocarcinoma by postoperative pathology (referring to the eighth edition tumor node metastasis [TNM] classification for NSCLC), and all had complete clinical data available with none receiving chemotherapy or radiotherapy prior to the operation. The hospital institutional review board of Kanazawa Medical University approved the protocol.

All enrolled patients were suggested to follow up according to our previous study protocol. The postoperative follow-up time points were every three months within the first year and every six months thereafter. During the follow-up, a physical examination, chest radiography, analysis of blood chemistry and carcinoembryonic antigen assay were performed. If any symptoms or signs of recurrence appeared in these examinations, further evaluations to detect the recurrent site were carried out. Follow-up time was terminated in May 2017 (median follow-up: 46 months).

\section{Histopathological evaluations}

Formalin-fixed, paraffin-embedded, 3- $\mu$ m-thick sections were obtained from 179 samples stained with hematoxylin and eosin for the histopathologic diagnosis. Of these, 144 cases had their pathological subtype and abundance ratio (over 5\%) from the maximal tumor diameter cross section recorded in the pathological report by two pathologists with a single blind method; the remaining cases lacking detailed pathological information were read again by the senior professor (Dr. Yamada) in order to obtain complete pathological data.

Lung adenocarcinomas were classified according to the IASLC/ATS/ERS Classification into the following subtypes: adenocarcinoma in situ (AIS), minimally invasive adenocarcinoma (MIA), lepidic (Lep), acinar (Aci), papillary (Pap), micropapillary (MP), solid (Sol) and mucinous (Mc) adenocarcinoma. Other relevant information was obtained from the surgical pathology report or our follow-up database.

\section{Pathological risk scoring system}

The final overall appraised ASP score of each specimen was determined as the pathological subtype points multiplied by the percentage content points. Adenocarcinoma in situ (AIS) was classified as a precancerous lesion and its 5-year overall survival rate was nearly $100 \%$. In its strictest sense, it was not belong to cancer. So we defined the pathological subtype points of AIS was 0 point. Minimally invasive adenocarcinoma (MIA) belong to adenocarcinoma mainly has a lepidic growth pattern, but its 
invasive component was less than $5 \mathrm{~mm}$. Furthermore, it had better prognosis as well as AIS. Therefore, the patients with MIA was diagnosed clinically, the pathological subtype points of MIA was defined as 1 point regardless of the invasive growth pattern. Other invasive adenocarcinomas include Lep, Aci, Pap, MP, Sol and Mc were given the different pathological subtype points according to their prognosis $[19,20]$. The worse the prognosis, the higher score. The specific scoring criteria and pattern are shown in Table 1 and Figure 1A. The distribution of the total score from 179 patients with stage I lung adenocarcinoma is shown in Figure $1 \mathrm{~B}$, and representative case contemporary contains three different growth patterns is shown in Figure 2A and 2B.

Table 1. Points assigned for different pathological subtypes and percentages for lung adenocarcinoma

\begin{tabular}{lllllll}
\hline Score & 0 point & 1 point & 2 point & 3 point & 4 point & 5 point \\
\hline $\begin{array}{l}\text { Pathological } \\
\text { subtypes }\end{array}$ & AIS & MIA & Lep & Aci\&Pap & Mc & MP\&So \\
$\begin{array}{l}\text { Percentages (\%) } \\
\text { 1 point }\end{array}$ & & & & & 1
\end{tabular}

Abbreviations: adenocarcinoma in situ (AIS), minimally invasive adenocarcinoma (MIA), lepidic adenocarcinoma (Lep), acinar adenocarcinoma (Aci), papillary adenocarcinoma (Pap), micropapillary adenocarcinoma (MP), solid adenocarcinoma (Sol) and mucinous adenocarcinoma (Mc).

A

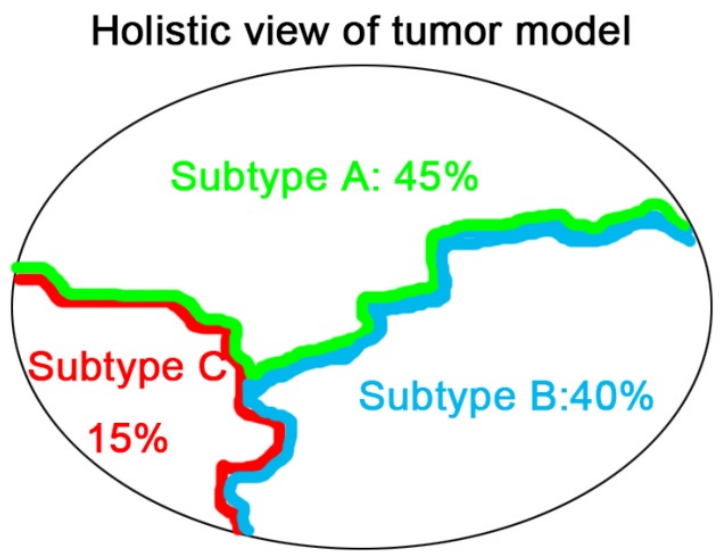

ASP scoring $=$ Pathological subtypes A scoring $\times(45 \% / 5 \%)$ Percentages scoring +

Pathological subtypes B scoring $\times(40 \% / 5 \%)$ Percentages scoring +

Pathological subtypes $\mathrm{C}$ scoring $\times(15 \% / 5 \%)$ Percentages scoring

B

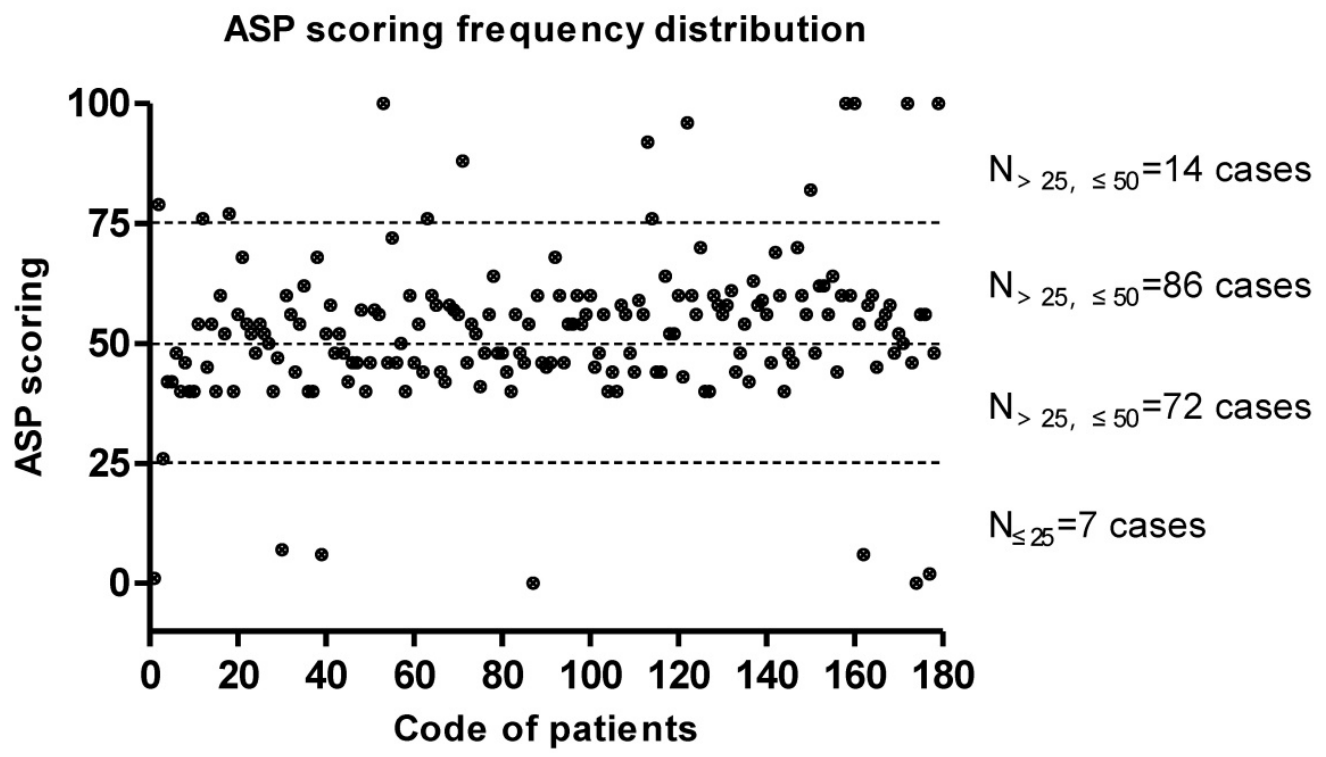

Figure 1. The Chart of ASP scoring pattern and its distribution in 179 patients with stage I lung adenocarcinoma. (A) A diagram of the formula on ASP scoring system. (B) The cumulative frequency curve chart of the ASP scoring distribution based on the pathological subtype and percentage content in 179 patients with stage I lung adenocarcinoma. The median ASP score was $52.7 \pm 16.3$ points. 
A

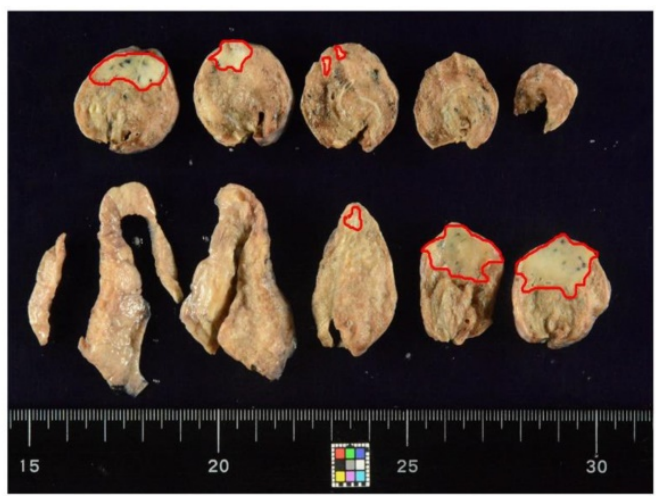

B

MP

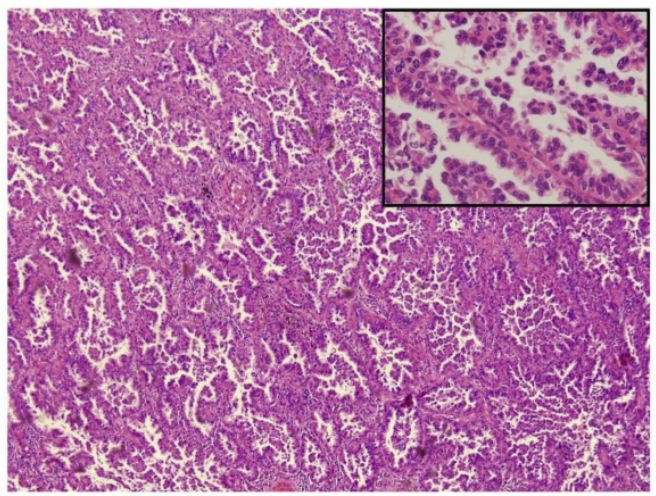

Pap

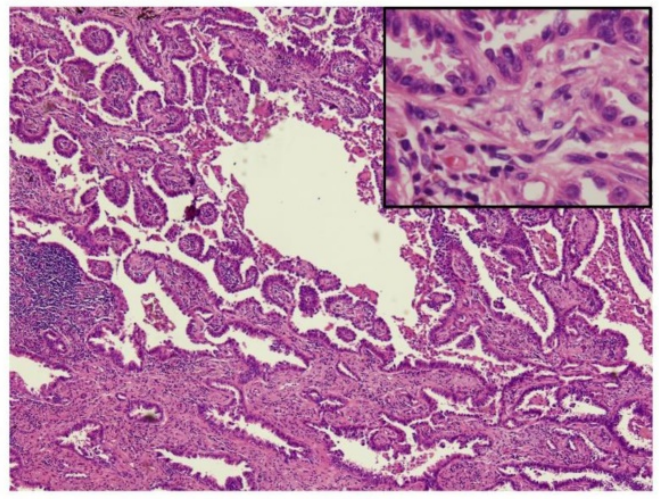

Lep

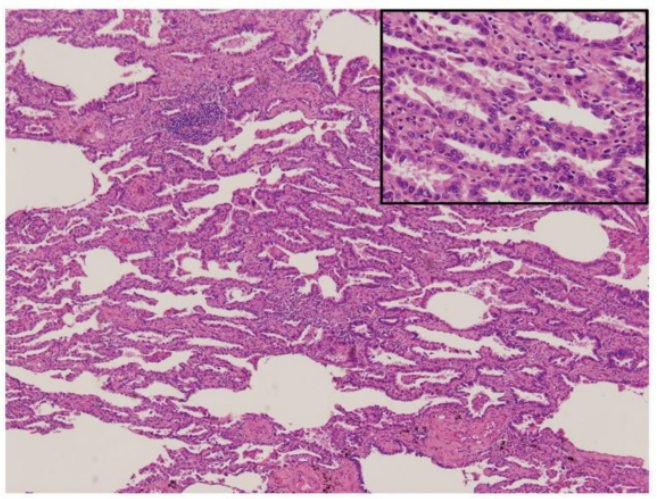

Figure 2. A typical case of mixed adenocarcinoma. (A) Focal area in the naked eye view, marked with a red dot. (B) The whole tumor contains lepidic (Lep; 40\%), papillary (Pap; 50\%), and micropapillary (MP; 10\%) growth patterns (magnification 40x and 100x). The ASP score of this patient was 2 points $\times(40 \% / 5 \%)+3$ points $\times$ $(50 \% / 5 \%)+5$ points $\times(10 \% / 5 \%)$

\section{Statistical analysis}

The association between the ASP score distribution interval and clinicopathologic characteristics was assessed by Pearson's chi-squared test. For the correlation analysis of the different ASP score and multiple variables, logistic regression analyses was constructed by the backward selection of multiple variables. Survival curves were calculated using the Kaplan-Meier method. The log-rank test was used to analyze the overall survival time by ASP scores in stage I lung adenocarcinoma. A univariate analysis was performed using the Cox regression model. Data were analyzed using the SPSS 22 software program (SPSS Inc, Chicago, IL, USA). Values of $\mathrm{P}<0.05$ were considered statistically significant.

\section{Results}

\section{Clinicopathologic characteristics correlation between the ASP score distribution and clinicopathologic variables}

A total of $87(48.6 \%)$ of the 179 patients with complete surgical resection of stage I lung adenocarcinoma were male, and $92(51.4 \%)$ were female. Among the 179 patients, 64 (35.8\%) were former or current smokers, and the smoking index exceeded 400 . In 66 patients (36.9\%), the expression of the tumor proliferation bio-marker Ki-67 exceeded $10 \%$. The numbers of cases in the right upper, right middle, right lower, left upper and left lower lobes were $60(33.5 \%), 20(11.2 \%), 33(18.4 \%), 40(22.4 \%)$ and $26(14.5 \%)$, respectively. The surgical procedure employed was open thoracotomy in 9 patients $(5.0 \%)$, complete video-assisted thoracic surgery [c-VAST] thoracotomy in 2 patients $(1.1 \%)$ and hybrid resection in 168 patients $(93.9 \%)$. Tumors were classified as well, moderately and poorly differentiated in 107 (59.8\%), 60 (33.5\%) and 12 (6.7\%) cases, respectively. Vascular and lymphatic invasion was observed in 48 (26.8\%) and $54(30.2 \%)$ cases, respectively.

\section{Correlation between the ASP score distribution and clinicopathologic variables}

Patients were divided based on their ASP score into a low-score group ( $\leq 50$ points; $\mathrm{n}=79[44.1 \%]$ ) and high-score group (>50 points; $\mathrm{n}=100$ [55.9\%]) (Table $1)$. The ASP score distribution was significantly associated with the smoking history $(p=0.004)$, lymphatic vessel invasion $(p<0.001)$, vascular invasion, differentiation $(\mathrm{p}<0.001)$ and Ki67 $(\mathrm{p}<0.001)$, as seen in Table 2, but not with the gender, age, location or maximum tumor diameter. Furthermore, patients with higher ASP scores tended to have poor differentiation, vascular or lymphatic invasion and 
abnormally elevated Ki67 expression, which often indicate a poor prognosis.

Subsequently, we found that ASP score categories were correlated with vascular invasion and high Ki67 expression using by logistic regression analysis. These values are shown in Table 3. In addition, we found that patients in the high-score group for ASP tended to have vascular invasion (odds ratio [OR]: 1.637, 95\% confidence interval [CI]: 1.923-13.745, $\mathrm{p}=0.001$ ) and high Ki67 expression (OR: 2.625, 95\%CI: 1.328-5.190, $\mathrm{p}=0.006)$ than those in the low-score group.

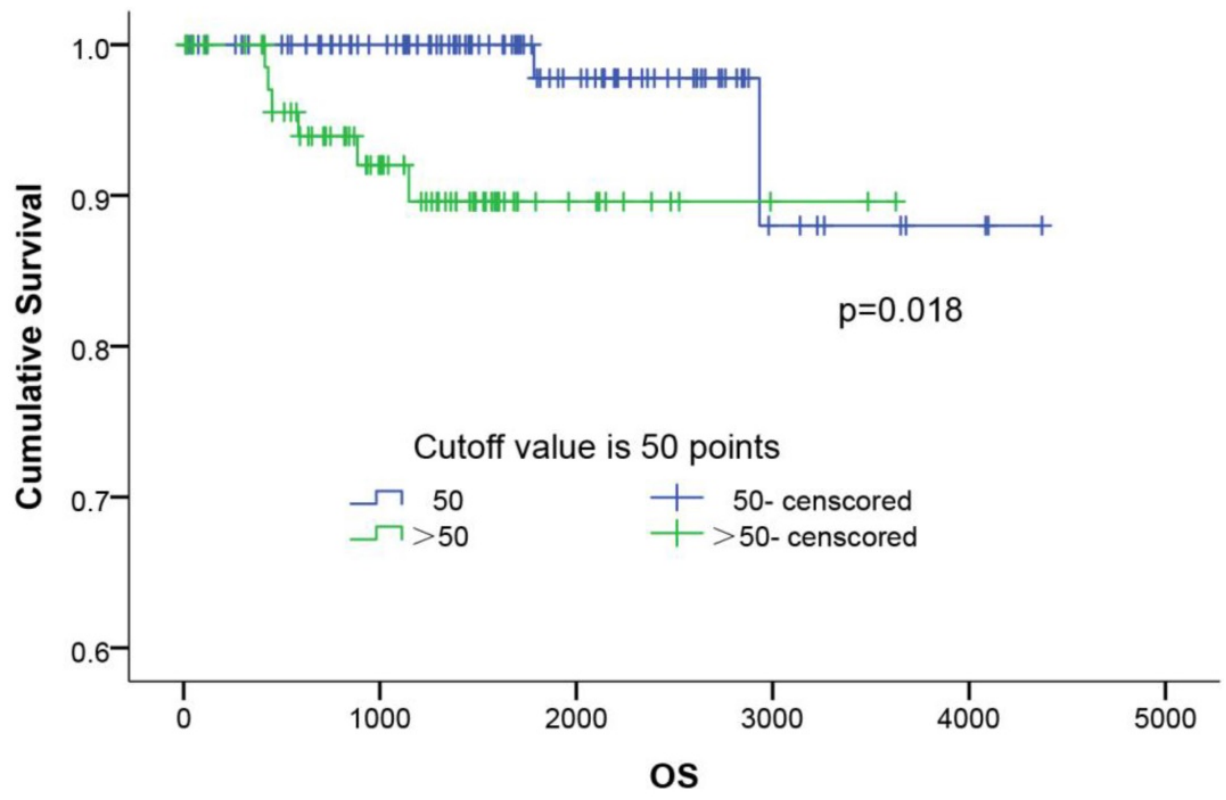

Figure 3. The survival curves of ASP scoring system. The survival curves of ASP scoring in 179 patients with completely resected stage I lung adenocarcinoma using the Kaplan-Meier method. The 5 -year survival rates of the low $(\leq 50)$, and high ASP score $(>50)$ groups was $97.8 \%$ and $89.6 \%$, respectively $(p=0.018)$.

Table 2. Relationship between the ASP scoring frequency distribution and clinicopathologic characteristics

\begin{tabular}{|c|c|c|c|c|c|c|c|}
\hline \multirow[t]{2}{*}{ Factor } & \multicolumn{3}{|c|}{ Low scores } & \multicolumn{3}{|c|}{ High scores } & \multirow[t]{2}{*}{$\mathrm{p}$-value } \\
\hline & $\leq 25$ & $>25$ to 50 & $\mathrm{~N} 1=79$ & $>50$ to 75 & $>75$ & $\mathrm{~N} 2=100$ & \\
\hline Gender & & & & & & & 0.054 \\
\hline male & 1 & 31 & $32(49.4 \%)$ & 44 & 11 & $55(50.6 \%)$ & \\
\hline female & 6 & 41 & 47 (64.1\%) & 42 & 3 & $45(35.9 \%)$ & \\
\hline Age, years & & & & & & & 0.341 \\
\hline$\leq 68.7$ & 3 & 39 & $42(47.7 \%)$ & 43 & 3 & $46(52.3 \%)$ & \\
\hline$>68.7$ & 4 & 33 & 37 (44.1\%) & 43 & 11 & $54(55.9 \%)$ & \\
\hline Smoking history & & & & & & & 0.004 \\
\hline$<400$ & 6 & 54 & $60(52.2 \%)$ & 53 & 2 & $55(47.8 \%)$ & \\
\hline$\geq 400$ & 1 & 18 & $19(29.7 \%)$ & 33 & 12 & $45(70.3 \%)$ & \\
\hline Location (lobe) & & & & & & & 0.674 \\
\hline right-up & 2 & 28 & $30(63.3 \%)$ & 26 & 4 & $30(36.7 \%)$ & \\
\hline right-mid & 1 & 6 & $7(50.0 \%)$ & 10 & 3 & $13(50.0 \%)$ & \\
\hline right-down & 2 & 11 & $13(45.5 \%)$ & 17 & 3 & $20(54.5 \%)$ & \\
\hline left-up & 0 & 19 & $19(67.5 \%)$ & 18 & 3 & $21(32.5 \%)$ & \\
\hline left-down & 2 & 8 & $10(46.2 \%)$ & 15 & 1 & $16(53.8 \%)$ & \\
\hline$T \max$ & & & & & & & 0.686 \\
\hline$\leq 1 \mathrm{~cm}$ & 4 & 1 & $5(71.4 \%)$ & 2 & 0 & $2(28.6 \%)$ & \\
\hline$>1 \mathrm{~cm}$ to $2 \mathrm{~cm}$ & 30 & 15 & 45 (58.4\%) & 20 & 12 & $32(41.6 \%)$ & \\
\hline$>2 \mathrm{~cm}$ to $3 \mathrm{~cm}$ & 14 & 17 & 31 (51.7\%) & 22 & 7 & $29(48.3 \%)$ & \\
\hline$>3 \mathrm{~cm}$ & 12 & 9 & $21(60.0 \%)$ & 4 & 10 & $14(40.0 \%)$ & \\
\hline Lymphatic vessel invasion & & & & & & & $<0.001$ \\
\hline Absent & 7 & 61 & $68(54.4 \%)$ & 47 & 10 & $57(45.6 \%)$ & \\
\hline Present & 0 & 11 & $11(20.4 \%)$ & 39 & 4 & $43(79.6 \%)$ & \\
\hline Vascular invasion & & & & & & & $<0.001$ \\
\hline Absent & 7 & 67 & $74(56.5 \%)$ & 50 & 7 & $57(43.5 \%)$ & \\
\hline Present & 0 & 5 & $5(10.4 \%)$ & 36 & 7 & $43(89.6 \%)$ & \\
\hline Differentiation & & & & & & & $<0.001$ \\
\hline High & 7 & 69 & $76(71.0 \%)$ & 30 & 1 & $31(29.0 \%)$ & \\
\hline Moderate & 0 & 3 & $3(5.0 \%)$ & 54 & 3 & $57(95.0 \%)$ & \\
\hline Poor & 0 & 0 & $0(0.0 \%)$ & 2 & 10 & $12(100.0 \%)$ & \\
\hline Ki67 & & & & & & & $<0.001$ \\
\hline$\leq 10 \%$ & 6 & 61 & $67(59.3 \%)$ & 43 & 3 & $46(40.7 \%)$ & \\
\hline$\geq 10 \%$ & 1 & 11 & $12(18.2 \%)$ & 43 & 11 & $54(81.8 \%)$ & \\
\hline
\end{tabular}


Table 3. Results of logistic regression analyses on patients with high or low ASP scoring and multiple variables

\begin{tabular}{llll}
\hline Variable & OR & $95 \%$ CI & p-value \\
\hline $\begin{array}{l}\text { Smoking history } \\
\geq 400\end{array}$ & 1 & & \\
$<400$ & 0.974 & $0.963-1.002$ & 0.312 \\
$\begin{array}{l}\text { Lymphatic vessel invasion } \\
\text { Present }\end{array}$ & 1 & & \\
$\begin{array}{l}\text { Absent } \\
\text { Vascular invasion }\end{array}$ & 0.571 & $0.526-3.200$ & 0.571 \\
$\begin{array}{l}\text { Absent } \\
\text { Present }\end{array}$ & 1 & & \\
$\begin{array}{l}\text { Differentiation } \\
\text { Moderate \& poor }\end{array}$ & 1.637 & $1.923-13.745$ & 0.001 \\
$\begin{array}{l}\text { High } \\
\text { Ki67 }\end{array}$ & 1 & & \\
$<10 \%$ & 0.885 & $0.638-1.229$ & 0.466 \\
$\geq 10 \%$ & 1 & & \\
\hline
\end{tabular}

Table 4. Univariate analysis of overall survival.

\begin{tabular}{|c|c|c|c|}
\hline Variable & OR & $95 \%$ CI & p-value \\
\hline \multicolumn{4}{|l|}{ Age, years } \\
\hline$\leq 68.7$ & 1 & & \\
\hline$>68.7$ & 12.489 & $0.349-43.794$ & 0.108 \\
\hline \multicolumn{4}{|l|}{ Gender } \\
\hline Male & 1 & & \\
\hline Femal & 0.111 & $0.014-0.906$ & 0.040 \\
\hline \multicolumn{4}{|l|}{ Smoking history } \\
\hline$<400$ & 1 & & \\
\hline$\geq 400$ & 7.250 & $1.452-36.195$ & 0.016 \\
\hline \multicolumn{4}{|l|}{ Operation method } \\
\hline c-VATS & 1 & & \\
\hline Hybrid\&Open & 3.465 & $0.635-18.891$ & 0.151 \\
\hline \multicolumn{4}{|l|}{ ASP scoring } \\
\hline Low & 1 & & \\
\hline High & 5.788 & $1.138-29.423$ & 0.034 \\
\hline \multicolumn{4}{|l|}{$\mathrm{T} \max$} \\
\hline$\leq 3 \mathrm{~cm}$ & 1 & & \\
\hline$>3 \mathrm{~cm}$ & 3.352 & $0.835-13.466$ & 0.088 \\
\hline \multicolumn{4}{|c|}{ Lymphatic vessel invasion } \\
\hline Absent & 1 & & \\
\hline Present & 0.380 & $0.046-3.116$ & 0.367 \\
\hline \multicolumn{4}{|l|}{ Vascular invasion } \\
\hline Absent & 1 & & \\
\hline Present & 1.283 & $0.254-6.493$ & 0.763 \\
\hline \multicolumn{4}{|l|}{ Differentiation } \\
\hline High & 1 & & \\
\hline Moderate\&Poor & 12.691 & $1.557-103.453$ & 0.018 \\
\hline \multicolumn{4}{|l|}{ Ki67 } \\
\hline$<10 \%$ & 1 & & \\
\hline$\geq 10 \%$ & 2.389 & $0.583-9.790$ & 0.226 \\
\hline
\end{tabular}

\section{Survival analyses}

The influence of the ASP score distribution on the patients' overall survival (OS) was evaluated. As shown in Figure 3, the Kaplan-Meier survival curves of all 179 patients showed that the patients with a higher ASP score $(\mathrm{P}=0.018)$ had a shorter OS than those with a lower score. The 5 -year survival rates in the low- $(\leq 50)$ and higher-ASP-score $(>50)$ groups were $97.8 \%$ and $89.6 \%$, respectively. Further, a multivariate Cox proportional hazards model analysis
(Table 4) of the stage I lung adenocarcinoma patients indicated that female (OR: 0.111, 95\% CI: 0.014-0.906, $\mathrm{p}=0.040$ ), long smoking history (OR: 7.250, 95\%CI: 1.452-36.195, $\mathrm{p}=0.016$ ), poor differentiation (OR: 12.691, 95\% CI: 1.557-103.453, $\mathrm{p}=0.018)$, and high ASP score (OR: 5.788, 95\%CI: 1.138-29.423, $\mathrm{p}=0.034$ ) were significant risk factors for predicting poor prognosis.

\section{Discussion}

In the present study, lung adenocarcinoma was frequently encountered in the form of a malignant mixed tumor, including two or more pathological subtypes, and these pathological subtypes showed obvious differences in tumor progression. This phenomenon, known as intra-tumor heterogeneity, may result in different prognoses among similar lung adenocarcinoma patients $[21,22,23]$. Indeed, Sica et al.[24] and Boland et al. [25] reported that the predominant combinations of pathological subtypes are associated with different prognoses. Although radiotherapy, chemotherapy and molecular-targeted therapy have improved the prognosis of patients with lung adenocarcinoma, $30 \%$ of patients with early lung adenocarcinoma still develop tumor metastasis or recurrence after undergoing radical surgery [26]. It has been suggested that the response to adjuvant therapy is unevenly distributed among these patients. Therefore, it is particularly important to distinguish patients at a high risk of recurrence and metastasis from seemingly identical patients who have a lower risk of such outcomes.

Many studies have shown that the assessed differing expression of tumor genes using immunohistochemical staining can effectively predict the prognosis in lung cancer [27, 28], but these biomarkers are only expressed in a certain subgroup of tumor cells and are measured according to pathologists' subjective experience. Furthermore, pathologists from different laboratories can draw opposing conclusions in the same case due to variations in sample processing and experimental techniques. Multilocus detection using secondgeneration sequencing technology has gradually developed [29, 30], but the costliness of such testing and low device penetration limit its application. In contrast, the approach proposed in the present study has advantages of simple controllability and good practicability. While this process may be timeconsuming and laborious, a new additional scoring formula on the pathological features (ASP) reveals crucial information on patients' lesions more comprehensively and accurately, thereby facilitating the differentiation of high-risk patients from lowerrisk ones and leading to appropriate early postoperative intervention, and which can be carried 
out smoothly in most primary-care hospitals. Furthermore, this scoring system also has prognostic implications, as it is capable of stratifying patients into different risk-rank groups for the OS of stage I lung adenocarcinoma and predicting the outcomes of individual patients after an operation. It therefore has potential utility in guiding disease management, similar to what is already being practicing in other organ systems.

Among the total 179 patients with stage I lung adenocarcinoma after complete surgical resection, patients with higher ASP scores tended to have poorly differentiation $(\mathrm{p}<0.001)$, vascular or lymphatic invasion $(\mathrm{p}<0.001)$ and abnormally elevated Ki67 expression $(\mathrm{p}<0.001)$, factors that are closely associated with a poor prognosis. This suggests that the ASP score may be more characteristic in evaluating patients' tumor status. Subsequently, the Kaplan-Meier survival curves also showed that the patients with higher ASP scores $(\mathrm{P}=0.018)$ had a shorter OS than those with lower scores. However, cytology or needle aspiration biopsy may not be appropriate for the ASP system that dependent on an adequately sampled resected tumor. While the nuclear morphology and chromatin mitotype usually in estimating of tumor malignant degree were not considered in this scoring system, but ASP was related to vascular invasion and Ki67 expression may indirectly reflect the degree of tumor malignancy to some extent. In the univariate analysis we found the significance of ASP score, but not appeared in the subsequent multivariate analysis (no listed in text). With the increased number of cases and the other clinical features, we expect the ASP system will be applicable not only to phase I lung cancer but also other stages of lung adenocarcinoma in the future. At present, technology supporting an artificial intelligence (AI) diagnosis is developing rapidly; [31, 32] such an approach may reduce the error-bias of supervisor judgment concerning the pathologic diagnosis and save labor costs. However, it may be some time before a mature algorithm and mathematics model can be established. As a shortterm solution, the ASP score system for assessing the prognosis of patients can be implemented in basiclevel hospitals, although it is admittedly timeconsuming and laborious to use. Our method of evaluating whole pathological sections may be used to generate references for an AI prototype algorithm. However, the disease-free survival is more significant than the overall survival we used in predicting the early recurrence and metastasis of lung adenocarcinomas, but we only obtained the cause of death and time of the patients via institutional investigation that was limitation of our study.
Therefore, the ASP scoring system needs to be further improved in the follow-up studies, including the acquisition of patients' disease-free survival.

\section{Conclusion}

In conclusion, the ASP score can effectively screen high-risk patients for complete surgical resection of stage I lung adenocarcinoma and help inform the early postsurgical management of these patients.

\section{Abbreviations}

IASLC: the International Association for the Study of Lung Cancer; ATS: the American Thoracic Society; ERS: the European Respiratory Society; ASP: additional scoring formula on the pathological features; AIS: adenocarcinoma in situ; MIA: minimally invasive adenocarcinoma; Lep: lepidic adenocarcinoma; Aci: acinar adenocarcinoma; Pap: papillary adenocarcinoma; MP: micropapillary adenocarcinoma; Sol: solid adenocarcinoma; Mc: invasive mucinous adenocarcinoma; AI: artificial intelligence.

\section{Acknowledgments}

We thank all doctors and staff of the Departments of Pathology and Thoracic Surgery from Kanazawa Medical University for their pathological assistance and direction.

\section{Author Contributions}

SL.Z. participated in its design, experimentation, analysis and manuscript drafting. X. G., and K. M., collected the clinical data and assisted in making the study design, and T. G., and FZ. L. evaluating the results obtained. S. Y. mainly performed histopathological examinations. H. U. contributed to theoretical organization of the manuscript. CD. G. and H. U. conceived and supervised the study, participated in its design, interpretation, and analysis, including drafting. SL.Z. and FZ. L. contributed equally to this study. All authors contributed to drafting and reviewing the manuscript and approved the submitted and final version.

\section{Ethics approval and consent to participate}

The study protocol was approved by the research ethics committee at the Kanazawa Medical University (I159). The need for written consent was waved by the Board because of the retrospective nature of the study. The research was conducted in accordance with the 1964 Declaration of Helsinki and its later amendments. 


\section{Consent for publication}

The datasets generated and analyzed during the current study are available from the corresponding author on reasonable request.

\section{Funding}

This work was supported by Grants-in-Aid for Scientific Research (No: 17K10803) from the Ministry of Education, Culture, Sports, Science and Technology, Tokyo, Japan; the National Natural Science Foundation of China (No: 81803886, 81774078).

\section{Competing Interests}

The authors have declared that no competing interest exists.

\section{References}

1. McIntyre A, Ganti AK. Lung cancer-a global perspective. J Surg Oncol. 2017; 115(5):550-554

2. Meza R, Meernik C, Jeon J, et al. Lung cancer incidence trends by gender, race and histology in the United States, 1973-2010. Plos One. 2015; 10(3):e0121323.

3. Williams MD, Sandler AB. The epidemiology of lung cancer. Cancer Treat Res. 2001; 105(105):31-52.

4. Hong QY, Wu GM, Qian GS, et al. Prevention and management of lung cancer in China. Cancer. 2015; 121(S17):3080-3088.

5. Reck M, Rabe KF. Precision diagnosis and treatment for advanced nonsmall-cell lung cancer. N Engl J Med. 2017; 377(9):849-861.

6. Chen Z, Fillmore CM, Hammerman PS, et al. Non-small-cell lung cancers: a heterogeneous set of diseases. Nat Rev Cancer. 2014; 14(8):535.

7. Zhang J, Fuijmoto J, Zhang J, et al. Intratumor heterogeneity in localized lung adenocarcinomas delineated by multiregion sequencing. Science. 2014; 346(6206):256-259.

8. Song $\mathrm{Z}$, Zhu $\mathrm{H}$, Guo $\mathrm{Z}$, et al. Prognostic value of the IASLC/ATS/ERS classification in stage I lung adenocarcinoma patients - Based on a hospital study in China. Eur J Surg Oncol. 2013; 39(11):1262-1268.

9. Woo T, Okudela K, Mitsui H, et al. Prognostic value of the IASLC/ATS/ERS classification of lung adenocarcinoma in stage I disease of J apanese cases. Pathol Int. 2012; 62(12):785-791

10. Yanagawa N, Shiono S, Abiko M, et al. New IASLC/ATS/ERS classification and invasive tumor size are predictive of disease recurrence in stage I lung adenocarcinoma. J Thorac Oncol. 2013; 8(5):612-618.

11. Detterbeck FC, Postmus PE, Tanoue LT. The Stage Classification of Lung Cancer: Diagnosis and Management of Lung Cancer, 3rd ed: American College of Chest Physicians Evidence-Based Clinical Practice Guidelines. Chest. 2013; 143(5):e191S-e210S

12. Travis WD, Brambilla E, Noguchi M, et al. Diagnosis of lung adenocarcinoma in resected specimens: implications of the 2011 International Association for the Study of Lung Cancer/American Thoracic Society/European Respiratory Society classification. Arch Pathol Lab Med. 2012; 137(5):685-705.

13. Borczuk AC. Prognostic considerations of the new World Health Organization classification of lung adenocarcinoma. Eur Respir Rev. 2016; 25(142):364-371.

14. Montserrat N, González A, Méndez E, et al. Impact of proposed IASLC/ATS/ERS classification of lung adenocarcinoma: prognostic subgroups and implications for further revision of staging based on analysis of 514 stage I cases. Modern Pathol. 2011; 24(5):653-664.

15. Russell PA, Zoe W, Wright GM, et al. Does lung adenocarcinoma subtype predict patient survival?: A clinicopathologic study based on the new International Association for the Study of Lung Cancer/American Thoracic Society/European Respiratory Society international multidisciplinary lung adenoc. J Thorac Oncol. 2011; 6(9):1496-1504.

16. Epstein JI, Egevad L, Amin MB, et al. The 2014 International Society of Urological Pathology (ISUP) consensus conference on Gleason grading of prostatic carcinoma. Am J Surg Pathol. 2016; 40(2):244-252.

17. Erdogan F, Demirel A, Polat Ö. Prognostic significance of morphologic parameters in renal cell carcinoma. Int J Clin Pract. 2004; 58(4):333-336.

18. Rakha EA, El-Sayed ME, Lee AH, et al. Prognostic significance of Nottingham histologic grade in invasive breast carcinoma. J Clin Oncol. 2008; 26(19):3153-3158.

19. Yoshiya T, Mimae T, Tsutani Y, et al. Prognostic role of subtype classification in small-sized pathologic N0 invasive lung adenocarcinoma. Ann Thorac Surg. 2016; 102(5):1668-1673.

20. Sica G, Yoshizawa A, Sima C S, et al. A grading system of lung Adenocarcinomas based on histologic pattern is predictive of disease recurrence in stage I tumors. Am J Surg Pathol. 2010;34(8):1155-1162.
21. De Sousa VML, Carvalho L. Tumor heterogeneity in lung cancer. Pathobiology. 2018;85(1-2):96-107.

22. De Bruin EC, Mcgranahan N, Swanton C. Analysis of intratumor heterogeneity unravels lung cancer evolution. Mol Cell Oncol. 2015; 2(3): e985549.

23. Xiao-Xiao S, Qiang Y. Intra-tumor heterogeneity of cancer cells and its implications for cancer treatment. Acta Pharmacol Sin. 2015; 36(10):1219-1227.

24. Gabriel S, Akihiko Y, Sima CS, et al. A grading system of lung adenocarcinomas based on histologic pattern is predictive of disease recurrence in stage I tumors. Am J Surg Pathol. 2010; 34(8):1155.

25. Boland JM, Wampfler JA, Yang P, et al. Growth pattern-based grading of pulmonary adenocarcinoma-Analysis of 534 cases with comparison between observers and survival analysis. Lung Cancer. 2017; 109:14.

26. Vansteenkiste I, Ruysscher DD, Eberhardt WEE, et al. Early and locally advanced non-small-cell lung cancer (NSCLC): ESMO Clinical Practice Guidelines for diagnosis, treatment and follow-up. Ann Oncol. 2013; 24 (Suppl 6): vi89-vi98

27. Gentles AJ, Newman AM, Liu CL, et al. The prognostic landscape of genes and infiltrating immune cells across human cancers. Nat Med. 2015; 21(8):938-945.

28. Inamura K. Lung Cancer: Understanding Its Molecular Pathology and the 2015 WHO Classification. Front Oncol. 2017; 7:193

29. Hagemann IS, Siddhartha D, Lockwood CM, et al. Clinical next-generation sequencing in patients with non-small cell lung cancer. Cancer. 2015; 121(4):631-639.

30. Paweletz CP, Sacher AG, Raymond CK, et al. Bias-corrected targeted next-generation sequencing for rapid, multiplexed detection of actionable alterations in cell-free DNA from advanced lung cancer patients. Clin Cancer Res. 2016; 22(4):915-922.

31. Chang HY, Jung CK, Woo JI, et al. Artificial Intelligence in Pathology. J Pathol Transl Med. 2019; 53(1):1-12.

32. Tizhoosh HR, Pantanowitz L. Artificial intelligence and digital pathology: Challenges and opportunities. J Pathol Inform. 2018; 14(9):38.

33. Dineen K M, Silvestri G A. The Epidemiology of Lung Cancer[M]// Malignant Tumors of the Lung. Springer Berlin Heidelberg. 2004. 